\title{
Procesos de enseñanza de las matemáticas en las instituciones educativas municipales de Chigorodo, Antioquia
}

\author{
Teaching processes of mathematics in the municipal educational \\ institutions of Chigorodo, Antioquia
}

Carmen Zunilda Córdoba Murillo

carcom-4@hotmail.com

Código ORCID: 0000-0002-1185-0575

Universidad Metropolitana Educación, Ciencia y Tecnología (Umecit),Ciudad de Panamá, Panamá

\section{Resumen}

La investigación tiene como propósito describir los procesos de enseñanza de las matemáticas que realizan los docentes de básica primaria de las instituciones educativas municipales de Chigorodó Antioquia. El estudio se desarrolló bajo una investigación descriptiva con un diseño de campo transeccional contemporáneo. La población la conformaron 102 docentes. Los datos se recolectaron mediante la técnica de la encuesta con un instrumento cuestionario para medir dicho evento. La validez se llevó a cabo a través de la técnica de juicio de expertos. Para el cálculo de la confiabilidad se utilizó el coeficiente de Alfa de Cronbach, el cual se calculó mediante el software SPSS19, el resultado obtenido fue de 0,85, considerándose el instrumento altamente confiable. El análisis de los datos se realizó mediante la estadística descriptiva, la frecuencia absoluta y porcentual y la medida de tendencia central mediana, por cuanto los datos se midieron en un nivel ordinal. Los resultados indican que los docentes desarrollan bajos procesos de enseñanza en sus clases de matemáticas.

\begin{abstract}
The purpose of the research is to describe the mathematics teaching processes carried out by elementary school teachers at the municipal educational institution of Chigorodó Antioquia. The study was developed under a descriptive investigation with a contemporary transectional field design. The population was made up of 102 teachers. The data were collected using the survey technique with a questionnaire instrument to measure said event. Validity was carried out through expert judgment technique. For the reliability calculation, the Cronbach's alpha coefficient was used, which was calculated using the SPSS19 software, the result obtained was 0.85, considering the instrument highly reliable. Data analysis was carried out using descriptive statistics, absolute and percentage frequency, and the median central tendency measure, since the data were measured at an ordinal level. The results indicate that teachers develop low teaching processes in their mathematics classes.
\end{abstract}

\author{
Palabras clave: \\ Enseñanza; \\ matemáticas; \\ planificación; \\ facilitación; \\ evaluación
}

\section{Keywords: \\ Teaching; mathematics; planning; facilitation; evaluation}




\section{INTRODUCCIÓN}

A lo largo de los años la educación fue y será un factor fundamental tanto para las personas como para la sociedad 1 en general, por una parte porque fija pautas de conductas $y$, por otra, porque forma parte de la identidad cultural y del desarrollo permanente de la sociedad y, por ende, de un país.

De igual manera, la Unesco (2010), considera la importancia de la educación cuando plantea:

una población educada tiene efectos positivos sobre otras áreas clave del desarrollo, tales como: menores tasas de mortalidad materna e infantil, reducción de las tasas de infección por VIH y SIDA... Además, los procesos educativos garantizan la construcción y transmisión de valores y actitudes comunes, favorecen la integración y participación de todos (s.n.)

Según Flórez (2002, p. XX), la educación se refiere, "a la interacción cultural, al proceso social mediante el cual una sociedad asimila a sus nuevos miembros incorporándolos a sus valores, reglas, pautas de comportamiento, saberes, prácticas, ritos y costumbre que la caracterizan". Así, la educación como acción socializadora ejerce cambios en la conducta del individuo, propiciándole inquietudes, conjeturas, espíritu crítico, que le conlleva al desarrollo de su talento, creatividad y su empoderamiento como persona humilde, responsable y honesta consigo misma y con los demás. De allí que la importancia y eficiencia de la educación, tal como lo plantea Pozo y Gómez (2001, 31), "deberá medirse por los que logremos que los alumnos aprendan realmente"

La educación involucra dos grandes procesos: la enseñanza y el aprendizaje. La enseñanza no puede entenderse más que en relación al aprendizaje, puesto que una verdadera enseñanza siempre tiene como fin el aprendizaje. Medina (2002, como se citó en Navarro, Rodríguez y Barcia (2011) define la enseñanza como:

el modo peculiar de orientar el aprendizaje y crear los escenarios más formativos entre docente y estudiantes, cuya razón de ser es la práctica reflexiva e indagadora, adaptando la cultura y el saber académico a los estudiantes en función de los valores educativos. (p. 40) 
Por su parte, Feldman (2010, p. 169), define el aprendizaje como "el cambio relativamente permanente en el comportamiento generado por la experiencia". El aprendizaje es el producto de la educación manifiesta por el sujeto, que es el objetivo de la educación.

En el caso particular del proceso de la enseñanza-aprendizaje de las matemáticas, la National Council of Teachers of Mathematics (N.C.T.M, 2000, como se citó en Godino y otros, 2003) considera que para que la enseñanza de las matemáticas sea efectiva requiere que los alumnos comprendan e internalicen qué conocimientos poseen y cuáles necesitan aprender, en razón de esto los estudiantes deben aprender las matemáticas "comprendiéndolas, construyendo activamente el nuevo conocimiento a partir de la experiencia y el conocimiento previo" (N.C.M.T, 2000, como se citó en Godino y otros, 2003, p. 12).

\section{Descripción de la situación problemática}

Según señala la Unesco (2015), entre los retos más relevantes que enfrenta el proyecto Educación para Todos está el buscar el modo más eficaz para que el estudiante adquiera competencias en matemáticas básicas para el paso al primer ciclo de secundaria, puesto que ésta es considerada como componente esencial para el acceso a la ciencia y la tecnología, con miras al desarrollo de otras habilidades de orden superior.

Por su parte, Godino, Batanero y Font (2003) consideran que "las matemáticas constituyen el armazón sobre el que se construyen los modelos científicos, toman parte en el proceso de modelización de la realidad, y en muchas ocasiones han servido como medio de validación de estos modelos" (p. 22).

En este contexto es obvio considerar que las aplicaciones matemáticas tienen una fuerte presencia en el quehacer del hombre, lo que permite inferir que el aprendizaje matemático es importante por cuanto propicia el desarrollo de habilidades que son garantía para el desenvolvimiento del estudiante en su contexto, así como un aporte fundamental para el acceso a la ciencia y la tecnología. Visto desde este contexto, potenciar el conocimiento matemático no solo es un reto de la educación a nivel global, sino que también lo es a nivel regional, donde la escuela, como ente que tiene la misión de velar y organizar el quehacer docente, asume la mayor responsabilidad. 
Con relación al sistema educativo colombiano, rural y lo urbano, este está divido en varias etapas, iniciándose con la educación inicial, la cual brinda atención a la primera infancia y es ofrecida a niños entre cero a cinco años de edad; luego, la educación básica, lapso de nueve años, divididos en: cinco años de educación primaria y cuatro años de secundaria, ofrecida a niños y jóvenes de entre seis y catorce años de edad; seguidamente la educación media, la cual comprende dos grados (décimo y undécimo), está prevista para jóvenes entre quince y diecisiete años de edad.

Finalmente, el ciclo del sistema educativo colombiano culmina con el sistema de educación superior, el cual incluye estudios técnicos, tecnológicos y profesionales. En este sistema no hay una edad promedio estipulada de ingreso, sin embargo, se espera que el estudiante al salir de la educación media, acceda a la educación superior y continúe su formación.

Los aspectos pedagógicos que sustentan el currículo de la educación inicial y educación básica del sistema educativo colombiano, están establecidos en la resolución 2343 de 1996, según los artículos 77, 78 y 148 de la Ley 115 de Educación (Ministerio de Educación Nacional/ MEN, 1994).

Sin embargo, dichos lineamientos curriculares estaban rezagados con respecto a los avances científicos-tecnológicos de la actualidad y, además, no establecían pautas o estrategias de aprendizaje para abordar disciplinas consideradas por el estudiante como "difíciles", particularmente, las matemáticas.

En razón de lo planteado y luego de intensas reflexiones del personal docente y directivos, el MEN declara los Lineamientos Curriculares para Matemáticas (1998), en este documento se plantean cambios ajustados a las nuevas realidades del contexto, que apunten hacia el aprendizaje matemático y le asignan carácter transversal.

Estos lineamientos curriculares reflejaban una postura pragmática e instrumental del conocimiento matemático, por lo que se consideraron una transición hacia la definición de las competencias básicas en matemáticas, las cuales son declaradas en 2006. Estas competencias conocidas como Estándares Básicos de Competencias en Matemáticas, tienen como visión, según el MEN (2006, p. 6) "que todos los estudiante reciban una educación de calidad y desarrollen las capacidades necesarias para enfrentar los retos del mundo contemporáneo". 
En consecuencia, incorporar las competencias en la formación del estudiante implica ir más allá del simple conocimiento; es despertar en el estudiante un desear hacer, ser y sentir, lo que involucra aspectos afectivos como la volición y la actitud; tal como lo plantean García y otros (2012, como se citó Solar, García, Rojas y Coronado (2014).

Sin embargo, a pesar de que en Colombia el desarrollo de las competencias en matemáticas tiene un asidero legal y curricular, se observa con preocupación que los resultados de pruebas como Saber se mantienen siempre por debajo del nivel mínimo establecido, lo que coloca al país en posiciones muy bajas en los resultados de las pruebas Pisa en áreas medulares del saber, como son lectura crítica, matemáticas y ciencia, e indican que la calidad de la educación en el país, no tiende a ser la mejor.

En efecto, al comparar los resultados de la prueba Saber (2018) se puede evidenciar que en el departamento de Antioquia el 52\% de los estudiantes se encuentran en nivel bajo con relación al país, el cual se ubica en un $44 \%$ en nivel bajo.; esto demuestra que los estudiantes de la entidad territorial (ET) presentan dificultades en el área de matemáticas, tales como: el análisis e interpretación de gráficas; poco manejo de resolución de problemas, dificultades para resolver fracciones y poco manejo de los procedimientos.

A partir de estos resultados, se infiere que el panorama actual de la educación en Colombia afianza la problemática de la que trata esta investigación, de igual modo se puede asegurar que la educación matemática, siendo una asignatura interdisciplinaria, es un referente importante para el acceso a las ciencias, puesto que permite el desarrollo de competencias en otras disciplinas

Igualmente, estas evidencias muestran, en cuanto a la calidad de la educación que las practicas a nivel de enseñanza continúan con el uso de técnicas y estrategias de tipo tradicional, lo cual propicia que el estudiante no desarrolla interés por el área lógicomatemáticas, siendo evidencia de esta problemática los resultados obtenidos en las pruebas antes citadas; además, limitan el desarrollo de su capacidad creativa, en tanto no procura el uso de otras estrategias, como las Tics.

Un estudio diagnóstico realizado en el año 2002, por parte de la Calvo, Rendón y Rojas (2004) de la Universidad Pedagógica Nacional, acerca de la formación de los docentes en Colombia, arroja preocupantes resultados, ya que sugiere que el docente en su 
práctica pedagógica suele soslayar el hecho de que el uso de las nuevas tecnologías responde a los cambios acelerados que está tomado la sociedad y que estos cambios, a su vez, tienen impacto en las diferentes culturas.

Por consiguiente, de nada vale al maestro de matemáticas saber todo acerca de éstas, si no sabe llevarlas al aula de clase y ponerlas accesibles al estudiante, es así como la utilidad de su saber adquiere pertinencia e importancia y se convierte en motivador de aprendizaje, a través del uso de herramientas que se alejen de la cátedra tradicional y se acerquen a aquello que el estudiante conoce, que vive diariamente y con lo que se ha familiarizado gradual y fácilmente, debido a los avances tecnológicos.

Cabe resaltar que la tecnología es una herramienta para acceder al conocimiento, así que la tarea de integrarla al proceso educativo subyace en la labor del maestro, quien de forma creativa está obligado a modificar su ejercicio, ahora bien, si no está familiarizado con las Tic la enseñanza es deficiente, lo cual genera: el desacato de sus estudiantes hacia el área, la desmotivación y el no desarrollo de las competencias requeridas para la promoción escolar, condición que se pone en evidencia durante la través del proceso de evaluación.

Con relación al proceso pedagógico, se observa que el docente utiliza para la enseñanza de la matemática una didáctica tradicional centrada en el tablero y ejercicios prácticos en el cuaderno. El maestro no utiliza ningún medio tecnológico acorde al contexto, lo cual genera desinterés y falta de motivación por parte del estudiante.

Así mismo, debido al poco acompañamiento que se le hace al estudiante, se presenta una incorrecta apropiación de los conceptos, igual deficiencia se da con relación a las operaciones entre fraccionarios, la potenciación y las propiedades que se cumplen en ellas, entre otros; contenidos considerados como necesarios para acceder al siguiente grado de educación básica secundaria. Todas estas debilidades son factores que propician el bajo rendimiento académico del alumno, tal como lo evidencian las pruebas Saber.

Cabe considerar que en la educación básica primaria, como consecuencia de la forma inadecuada de abordar dichos contenidos programáticos, se comienzan a presentar las falencias que conllevan a la no apropiación de las temáticas, el no desarrollo de 
las competencias propias del grado, lo cual impide que se haga el encadenamiento de los conocimientos propios de dichas temáticas, condición que se considera absolutamente necesaria para continuar en el siguiente nivel.

De acuerdo a lo anteriormente planteado se formula como pregunta de investigación la siguiente: ¿Cómo son los procesos de enseñanza de las matemáticas que desarrollan los docentes de las instituciones educativas municipales de Chigorodo, Antioquía?

\section{Proceso de enseñanza-aprendizaje}

En el proceso de enseñanza-aprendizaje, conformado por la planificación, la facilitación y la evaluación, intervienen el maestro como gestor y el estudiante como constructor, ambos confluyen en el escenario que implica la escuela. El maestro, como partícipe del proceso en calidad de orientador y guía, ha de entender que el aprendizaje en los alumnos se manifiesta de diversas formas, es decir, todos no aprenden igual y al mismo ritmo, por consiguiente el trabajo del pedagogo, consiste en guiar esos procesos de acuerdo a las necesidades del estudiante.

Flórez (2002, p. XXII), considera que "la enseñanza es una actividad educativa más específica, intencional y planeada para facilitar que determinados individuos se apropien y elaboren con creatividad cierta porción del saber o alternativas de solución a algún problema en aras a su formación personal". Por su parte, Quesada (1991, p. 16) define el aprendizaje como un proceso en el cual se da "la internalización de pautas de conducta que resulta de haber participado en un proceso intencionado de enseñanzaaprendizaje".

Los conceptos de enseñanza y aprendizaje son dos términos correlacionados, inseparables el uno del otro, relacionados por causa y efecto al considerar que se enseña porque existe la necesidad del aprender, por lo tanto no puede caracterizarse a la enseñanza desvinculándola del aprendizaje, razón por la cual Hirst (1977, p. 304) señala "No existe nada a lo que podamos llamar enseñanza, sin la intención de dar lugar un aprendizaje. Así pues, hasta que no sepamos qué es el aprendizaje, no sabremos qué es enseñar. Uno de estos conceptos es totalmente dependiente del otro".

Si bien es cierto que algunas veces el proceso de enseñanza no se ve cristalizado en aprendizaje, también lo es que la función de la 
enseñanza no es producir per se aprendizaje, puesto que aprender es un proceso que depende propiamente del individuo; el propósito de la enseñanza es intervenir en el aprendizaje y proporcionar un ambiente propicio para que el estudiante relacione de manera sustantiva y no arbitraria los nuevos conocimientos con los preexistentes.

Según Matus (1987 como se citó en Samper, 2012, p. 17), “... planificar es un proceso de análisis y discusión para seleccionar una dirección que guíe la transformación hacia la situación deseada". De igual manera, De acuerdo a Zabalza (2003, como se citó en Ercolino y Morales, 2006, p. 6), "la capacidad de planificar conforma una de las primeras competencias docentes del profesor; por lo que la mejora de la enseñanza depende en gran parte de la calidad y actualización pedagógica de esta competencia por parte del educador"

Ante lo planteado, se infiere que la planificación en el proceso enseñanza- aprendizaje consiste en la ruta que fija el docente durante el proceso de enseñanza y que le permite reflexionar sobre cómo abordar su hacer pedagógico. Establecer esta ruta comprende darle respuesta a interrogantes tales como: qué hacer, por qué, dónde y cómo lo hará, con qué cuenta y cómo hacer para saber que ha logrado los objetivos planteados. Es decir, planificar implica trazar un plan de acción sobre qué se enseñará y cómo se enseñará considerando los conocimientos previos que posee el estudiante.

Los elementos involucrados en la planificación del proceso de enseñanza-aprendizaje son: los objetivos, los contenidos, las actividades didácticas, las estrategias de aprendizaje, los medios y los criterios de evaluación.

Según Salcedo (2011, p. 116), los objetivos son "formulaciones de carácter didáctico que expresan en forma clara y precisa los cambios de conducta que se han de operar en el alumno como efecto del proceso enseñanza-aprendizaje". Por medio de la planificación el docente de manera anticipada fija los objetivos de logro del estudiante, y a partir de allí establece los contenidos, estrategias y medios para alcanzar tal fin, así como los criterios que le permitirán evaluar el nivel de logro.

Los contenidos en el proceso de enseñanza-aprendizaje constituyen toda la información suficiente y necesaria planificada por el docente para que el estudiante alcance un aprendizaje significativo. 
Las actividades didácticas, según Cañal y otros (1997, 121), "son procesos de flujo y tratamiento de información (orientados, interactivos y organizados) característicos del sistema-aula". Se consideran orientados porque se direccionan en función de las metas didácticas planteadas, interactivos porque establecen la intercomunicación entre docentes-alumnos-contexto y organizados porque se manejan mediante condiciones preestablecidas.

Los medios didácticos se constituyen en herramientas de gran apoyo al personal docente como al estudiante en el proceso de enseñanza-aprendizaje, entre los beneficios que generan se tienen: facilitan la adquisición del conocimiento, motivan y despiertan el interés, ilustran y focalizan lo que el docente expone al estudiante, facilitan la comprensión del contenido expuesto, así la fijación del aprendizaje, entre otras.

Con respecto a los criterios de evaluación, se consideran que estos son normas concretas y claramente definidas que permiten emitir un juicio o tomar una decisión respecto al nivel de aprendizaje del estudiante. Según Alfaro (2000, como se citó en Camacho 2014, p. 46) los criterios de evaluación "son los puntos de referencia con los cuales se va a evaluar, "los resultados del aprendizaje, calidad, valores y creencias que se han ido formando en el proceso de enseñanza aprendizaje".

El gran reto del docente se centra en desarrollar una planificación coherente con su concepción pedagógica, que, a lo largo de su desempeño desarrolle estrategias que permitan a sus estudiantes incorporar nuevos conocimientos a sus esquemas mentales para conformar aprendizajes significativos. Una herramienta valiosa para este fin lo constituye la habilidad que pueda manejar el docente para facilitar el aprendizaje a sus alumnos a través de diferentes estrategias, técnicas, métodos o procedimientos, entre otros.

Sin embargo, la labor docente muchas veces resulta insatisfactoria, puesto que ocasionalmente se cuenta con las herramientas necesarias para desarrollar tan valiosa misión, no obstante, el trabajo del docente consiste, precisamente, en llevar a cabo la labor con lo que posea y con lo que sea susceptible de implementar, ya que según Vygotsky (1978) los alumnos aprenden mejor en colaboración con sus pares profesores, padres y otros, cuando se encuentran involucrados de forma activa en tareas significativas e interesantes. 
Por su parte, García, Coronado y Montealegre (2011, p. 20) plantean que "una forma de lograr un resultado positivo en el proceso formativo escolar es hacer del contenido una interacción con el mundo real", lo cual implica que el maestro debe llevar a cabo acciones facilitadoras que vayan encaminadas a que el estudiante adquiera las habilidades que son el objetivo formativo escolar.

Según Arce (2012), la facilitación es una gestión de procesos y funciones a objeto de que un grupo humano pueda lograr sus objetivos en un ambiente agradable con el buen uso de los recursos y el tiempo. En este contexto, la facilitación persigue el abordaje de la enseñanza con estrategias tales que permitan la realización del proceso de aprendizaje de manera fácil y sencilla; además de fomentar y desarrollar, con una gran fuerza liberadora, las capacidades y energías internas de los individuos.

Un efectivo proceso didáctico de facilitación orientado hacia los estudiantes requiere, fundamentalmente, que se establezcas niveles de confianza y credibilidad en sus habilidades y destrezas, (Arnold 2016). En este sentido, según Padilla, Rincón y Lagos (2016):

La facilitación del aprendizaje se sustenta en una transformación de la pedagogía tradicional enmarcada en la enseñanza, a una cambiante forma de incluir al educando de cara activa al proceso pedagógico, en la cual el docente al igual que el estudiante desarrolla procesos de construcción de aprendizaje y enseñanza. $(\mathrm{s} / \mathrm{n})$

En efecto, en el marco de la enseñanza centrada en la interacción facilitador-alumno, el docente direcciona su atención hacia el estudiante, cambia el rol de "el que sabe y transmite" por el que acompaña, guía y orienta "en la aventura de descubrimiento que supone el aprendizaje" (Ochoa y Ochoa, 2016, p. 75). Este nuevo accionar didáctico redefine el papel del docente $y$, en consecuencia, las nuevas alternativas para su formación y desarrollo profesional.

En razón de esta nueva visión, entre el facilitador que enseña y el alumno que aprende se establece una relación dialéctica (Freire, 1973). Esta bidireccionalidad hace que dicho proceso sea interactivo y, a su vez, fomenta una pedagogía dialógica, la cual 
ayuda a los alumnos en la construcción de su propio aprendizaje a través una realidad vinculada con el conjunto de experiencias y conocimientos previos utilizados para construir nuevos saberes denominado aprendizaje significativo.

En razón de lo planteado se infiere que el alumno solo aprende cuando el conocimiento que se le ofrece es relevante para él, es decir, su aprendizaje está en función del sentido que tenga para él lo que aprende. Este tipo de aprendizaje se diferencia sustancialmente del aprendizaje memorístico, el cual, como su nombre lo refiere, se centra en la simple memorización, sin que el alumno logre integrar los nuevos conocimientos adquirido con conocimientos previos relevantes en su estructura cognoscitiva.

Con relación al proceso de enseñanza-aprendizaje de las matemáticas, puede considerarse que por mucho tiempo ha ocupado largas horas de reflexión en los individuos vinculados con la didáctica, en virtud de considerarse en el ámbito educativo el área lógico-numérico fundamental para que el estudiante alcance el manejo de destrezas que tienen múltiples aplicaciones (el razonamiento, el pensamiento lógico y creativo, la argumentación fundamentada, entre otros) en su hacer diario, desde su fase de niño hasta su vida profesional, aunado a la importancia que reviste para el país desde el punto de vista económico, político y social.

La didáctica, con el apoyo de las teorías de la enseñanzaaprendizaje, facilita al docente el manejo de diversos medios para abordar la enseñanza de las matemáticas, utilizándose desde los más elementales como es el lenguaje natural hasta los artificiales como la computadora y sus respectivos.

Sin embargo, periódicamente los resultados que se obtienen en las aulas de clase con respecto a las disciplinas con componentes de aplicación lógico-numéricos no son muy alentadores, tal como se demostró en párrafos anteriores. Este planteamiento demanda de los docentes ocupados en la enseñanza, y muy particularmente en la disciplina de las matemáticas, una revisión continúa de su hacer pedagógico a fin de aplicar los métodos, herramientas y estrategias más idóneas que garanticen el aprendizaje de los estudiantes, tal como lo refiere Orealc/Unesco. (2014)

Para mejorar el aprendizaje de todos los niños, los docentes necesitan apoyarse en estrategias en materia de planes y programas de estudios y en evaluaciones que 
permitan reducir las disparidades en el aprovechamiento escolar y ofrecer a todos los niños y jóvenes la oportunidad de adquirir competencias transferibles esenciales. Estas estrategias deben servir para desarrollar sólidas competencias básicas, comenzando tempranamente y avanzando a un ritmo adecuado, permitiendo a los alumnos desfavorecidos ponerse al día (p. 11)

En ese orden de ideas, es pertinente potenciar el logro de los aprendizajes matemáticos con metodologías que atiendan herramientas didácticas que generen en el estudiante la suficiente motivación capaz de lograr su propio aprendizaje al encontrarle sentido, pertinencia y aplicación a lo que aprende.

Al tratar el proceso de enseñanza-aprendizaje es imposible no abordar el proceso de evaluación del conocimiento, puesto que a través de la evaluación se logra obtener información válida, confiable y oportuna sobre los logros alcanzados por el estudiante con relación al proceso de aprendizaje, con el fin de emitir un juicio de valor que permita tomar diversos tipos de decisiones.

La definición del término evaluación muestra muchas conceptualizaciones, algunas relacionadas, particularmente, con juicios de valor sobre objetivos de enseñanza, instrumentos, medición, calidad del programa e institución; otras centradas en establecer la calidad de la enseñanza brindada y el progreso del estudiante. En razón de esto, se considera importante que cada docente tenga muy claro su propia definición de evaluación pues ella servirá para determinar la calidad del aprendizaje.

Ahora bien, es obvio que el docente define y aplica en su práctica la evaluación en función de las teorías que estructuran su pensamiento pedagógico; de la concepción didáctica que internalizó durante su proceso de formación, de sus motivaciones, así como de la responsabilidad con la que asume su hacer pedagógico. Pues tal como lo refiere Zapata (2005, como se citó en Ortiz, 2009, p. 92), "las actividades de enseñanza y evaluación están guiadas por series de creencias o amalgamas de creencias y supuestos sobre qué son y cómo se producen"

Por su parte, Santos (1993) plantea que la evaluación sólo facilita la mejora del proceso enseñanza-aprendizaje si se logra considerar el valor educativo del programa, al facilitar la comprensión de lo que sucede en el mismo y permitir medir los niveles de aprendizaje a nivel educativo. 
En ese orden de ideas, para Tobón (2010, p. 36) la evaluación "es un procedimiento para determinar los avances de los estudiantes en la obtención de los conocimientos establecidos en el currículo".

Hoy día el proceso de evaluación del aprendizaje enmarcado en la visión constructivista permite conocer qué sabe el estudiante o que es capaz de hacer; proporciona retroalimentación a los estudiantes acerca de los que requieren para mejorar sus saberes. De igual manera, brinda un "feedback" al docente sobre cómo desarrolla su proceso de enseñanza, lo cual le permite identificar las debilidades y realizar los ajustes necesarios con el propósito de alcanzar una mejorar calidad en el proceso de evaluación.

Los resultados de dichos instrumentos deben ser considerados como indicios que lleven a reflexionar, por una parte, al docente sobre la manera como desarrolla su proceso de enseñanza y evaluación y, qué aspectos pudiera mejorar a fin de que despierten el interés y la motivación de aprender a aprender en sus estudiantes; por la otra a los estudiantes, que se conciban personas reguladoras de su propio aprendizaje, que asumen la evaluación con una actitud positiva y como estrategia permanente de mejora de sus saberes. A la institución, su reflexión se centra en la revisión de las políticas educativas que rigen el modelo pedagógico que aplica.

El proceso de evaluación tiene diferentes propósitos, tales como obtener información para tomar decisiones administrativas, información para el estudiante sobre su desempeño académico, información para el docente sobre el proceso de enseñanza que desarrolla, pronóstico sobre el desempeño de los estudiantes, entre otros.

La evaluación del proceso de enseñanza-aprendizaje puede clasificarse según varias modalidades. Siendo la modalidad más aplicada la referida al momento en que se realiza la evaluación. Según López y Hinojosa (2011) este tipo de evaluación se clasifica en: evaluación inicial o diagnóstica, evaluación formativa y evaluación sumativa. Cada momento de evaluación tiene distintos objetivos. 


\section{METODOLOGÍA}

$\mathrm{L}$

a presente investigación se consideró de tipo descriptiva, con

un diseño de campo, transeccional contemporánea, con amplitud de foco unieventual. El proceso de recolección de datos sobre los procesos de enseñanza de las matemáticas de los estudiantes del grado quinto de las instituciones educativas municipales de Chigorodó Antioquia, se llevó a cabo a través de la técnica de la encuesta mediante un instrumento cuestionario para medir dicho evento. La validez se llevó a cabo a través de la técnica de juicio de expertos. Para el cálculo de la confiabilidad se utilizó el coeficiente de Alfa de Cronbach, el cual se calculó mediante el software SPSS19, el resultado obtenido fue de 0,85, lo cual hace el instrumento altamente confiable. La población objeto de estudio la conformaron 102 docentes de básica primaria de la institución educativa José de los Santos Zúñiga. Para el análisis de los datos referidos a los procesos de enseñanza se utilizó la estadística descriptiva, la frecuencia absoluta y porcentual y la medida de tendencia central mediana, por cuanto los datos se midieron en un nivel ordinal. Para la interpretación de los resultados se utilizó el baremo de interpretación que se muestra en el tabla 1.

Tabla 1. Baremo de interpretación de procesos de enseñanza

\begin{tabular}{cl}
\hline Rango & \multicolumn{1}{c}{ Categoría } \\
\hline $0-9,99$ & Muy bajos procesos de enseñanza \\
$10-19,99$ & Bajos procesos de enseñanza \\
$20-29,99$ & Medianos procesos de enseñanza \\
$30-39,99$ & Altos procesos de enseñanza \\
$40-50$ & Muy altos procesos de enseñanza \\
\hline
\end{tabular}

Fuente: Elaboración propia 


\section{Resultados de la investigación}

\section{Descripción del evento procesos de enseñanza}

En la tabla 2 se presentan los resultados del evento procesos de enseñanza, en esta se observa una mediana de 14,09 puntos sobre 50 puntos, lo cual indica que los docentes desarrollan bajos procesos de enseñanza de las matemáticas, es decir, los docentes no conocen sobre los aspectos básicos o manera de desarrollar un proceso de enseñanza.

Tabla 2. Estadísticos de procesos de enseñanza

\begin{tabular}{llr}
\hline N & Válidos & 102 \\
Mediana & & 14,09 \\
Mínimo & & 13 \\
Máximo & & 21 \\
Percentiles & 25 & 13,18 \\
& 50 & 14,09 \\
& 75 & 21,36 \\
\hline
\end{tabular}

Fuente: Elaboración propia (2020)

En la tabla 3 se presenta la distribución en las categorías de procesos de enseñanza, en el mismo se observa que $70,6 \%$ del grupo de docentes presenta bajos procesos de enseñanza de las matemáticas, por lo que se puede afirmar que son docentes que enseñan las matemáticas desde lo tradicional, con técnicas y actividades centradas en la ejercitación y limitan el uso de recursos a solo el tablero y el cuaderno o libro de ejercicios.

Tabla 3. Categorías de procesos de enseñanza

\begin{tabular}{|c|c|c|c|c|c|}
\hline & & Frecuencia & Porcentaje & $\begin{array}{l}\text { Porcentaje } \\
\text { válido }\end{array}$ & $\begin{array}{l}\text { Porcentaje } \\
\text { acumulado }\end{array}$ \\
\hline \multicolumn{6}{|l|}{ Válidos } \\
\hline & $\begin{array}{l}\text { Bajos procesos de } \\
\text { enseñanza }\end{array}$ & 72 & 70,6 & 70,6 & 70,6 \\
\hline & $\begin{array}{l}\text { Medianos procesos } \\
\text { de enseñanza }\end{array}$ & 30 & 29,4 & 29,4 & 100,0 \\
\hline & Total & 102 & 100,0 & $\begin{array}{r}100, \\
0\end{array}$ & \\
\hline
\end{tabular}

Fuente: Elaboración propia (2020) 


\section{Análisis de las sinergias de procesos de enseñanza}

Las sinergias del evento procesos de enseñanza están referidas a las acciones que el docente realiza en su enseñanza, siendo estas: la planificación, facilitación y evaluación, comportamiento que se muestra en la tabla 4.

Tabla 4. Estadísticos de las sinergia de procesos de enseñanza

\begin{tabular}{|c|c|c|c|c|}
\hline & & $\begin{array}{c}\text { Puntaje } \\
\text { transformado } \\
\text { planificación }\end{array}$ & $\begin{array}{c}\text { Puntaje } \\
\text { transformado } \\
\text { facilitación }\end{array}$ & $\begin{array}{c}\text { Puntaje } \\
\text { transformado } \\
\text { evaluación }\end{array}$ \\
\hline $\mathrm{N}$ & & 102 & 102 & 102 \\
\hline \multicolumn{5}{|l|}{ Válidos } \\
\hline Mediana & & 10,71 & 20,00 & 20,83 \\
\hline Mínimo & & 9 & 15 & 15 \\
\hline Máximo & & 20 & 25 & 21 \\
\hline \multirow[t]{3}{*}{ Percentiles } & 25 & 8,93 & 15,00 & 14,58 \\
\hline & 50 & 10,71 & 20,00 & 20,83 \\
\hline & 75 & 19,64 & 25,00 & 20,83 \\
\hline
\end{tabular}

Fuente: Elaboración propia (2020)

\section{Sinergia planificación}

Con respecto a la sinergia planificación, la tabla 4 muestra una mediana de 10,71 puntos sobre 50 puntos, valor que corresponde según el baremo de interpretación a la categoría de bajos procesos de planificación y lo cual indica que los docentes desarrollan durante el proceso de enseñanza de las matemáticas bajos procesos de planificación de las actividades académicas. De igual manera, el puntaje mínimo de 9 puntos expresa con claridad que algunos docentes solo cumplen con el $18 \%$ de los requerimientos para una planificación de la enseñanza. Es obvio que estos resultados reflejan que los docentes improvisan las clases y no llevan una planeación organizada que busque el desarrollo de competencia en los estudiantes.

Asimismo, la distribución de las categorias ubicó al 30,4\% de los docentes en la categoria muy bajos procesos de planificación, en tanto que el $69,6 \%$ en bajos procesos, lo cual coincide con la mediana obtenida.

Por otra parte, al realizar el análisis de ítems para el proceso de planificación se observó que los aspectos con más dificultades para los docentes fueron los referidos a: 
Los objetivos que relacionan la interacción de los estudiantes con el contenido de las matemáticas, la relación de los objetivos con la evaluación de competencias matemáticas apoyadas en medios tecnológicos, la selección del material didáctico que permita la realización y ejecución de ejercicios de aplicación, el uso de medios tecnológicos para dar explicación a los temas matemáticos del grado, el diseño de actividades individuales y por equipos de trabajo donde el contenido vaya acompaño de la explicación y de un ejercicio de aplicación matemática. Así como dificultad en la selección de material didáctico, de contenido teórico, representaciones y ejemplos resueltos, entre otros.

\section{Sinergia facilitación}

Según la tabla 4 la facilitación presenta una mediana de 20 puntos de un máximo de 50 puntos, la cual al ser comparada con el baremo de interpretación indica que los docentes desarrollan medianamente el proceso de facilitación, el cual está referido a las acciones que realizan durante la clase, es decir, la explicación y las técnicas, así como las actividades que desarrollan en la enseñanza.

Con relación a las categorías de facilitación se determinó que un $40,2 \%$ se ubicó en bajos procesos y $59,8 \%$ en medianos procesos, lo cual refleja que los docentes encuestados dictan las clases mediante técnicas y actividades que no logran conectar los conocimientos nuevos con los previos y por lo tanto no se logra el aprendizaje de la asignatura.

Con respecto a los resultados arrojados en el análisis de ítems para el proceso de facilitación se observó que los aspectos con más dificultades para los docentes fueron los referidos a:

La utilización de los materiales diseñados en la planificación para la clase, las explicaciones de los contenidos matemáticos con ayuda de las Tics, las explicaciones en la resolución de los ejercicios, la relación del contexto con los ejercicios matemáticos, la interacción de los estudiantes y los medios tecnológicos, la explicación de las actividades desarrolladas en la planificación, la explicación de la solución de ejercicios matemáticos, la participación de los estudiantes en la aplicación de ejercicios matemáticos. 


\section{Sinergia evaluación}

En cuanto a la sinergia evaluación, lo resultados obtenidos reflejan una mediana de 20,83 (tabla 4), lo cual indica que los docentes de matemáticas encuestados desarrollan medianamente actividades de evaluación referidas al desarrollo de pruebas y retroalimentación de los estudiantes de acuerdo a los requerimientos de la asignatura.

Con respecto a las categorías de la sinergia evaluación se observó que los docentes se distribuyen en un $30,4 \%$ en bajos procesos y 69,6 \% en medianos; ante estos resultados puede afirmarse que los docentes encuestados despliegan bajos procesos en la evaluación de la enseñanza, es decir, no aplican estrategias que les permitan identificar el nivel de avance de los estudiantes en su proceso académico.

Asimismo, el análisis de ítems para el proceso de evaluación permitió identificar los aspectos con más dificultades para los docentes, estos fueron:

La evaluación de forma grupal, para medir el trabajo en equipo y colaborativo; el desarrollo de pruebas individuales para medir los conocimientos de los estudiantes; las evaluaciones en línea; la elaboración de talleres; la utilización de recursos tecnológicos en forma contextualizada; las sugerencias de los estudiantes sobre algunas rutas de solución de los ejercicios; la aclaración de dudas con la participación de los estudiantes de la clase y con diferentes estrategia.

\section{DISCUSIÓN}

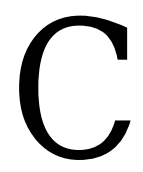

on relación a los procesos de enseñanza de los docentes de básica primaria de la institución educativa municipal José de los Santos Zúñiga del Chigorodó-Antioquia demostraron que estos desarrollan bajos procesos de enseñanza en sus clases de matemáticas, es decir, los docentes no realizan de manera efectiva y organizada la planificación, facilitación y evaluación en su quehacer educativo. Esta afirmación se apoya en los resultados obtenidos donde se evidenció que un 70,6\% del grupo de docentes se ubicaron en la categoría de bajos procesos.

Estos resultados resultan antagónicos a lo planteado por Flórez (2002, p. XXII), cuando considera que "la enseñanza es una 
actividad educativa más específica, intencional y planeada para facilitar que determinados individuos se apropien y elaboren con creatividad cierta porción del saber o alternativas de solución a algún problema en aras a su formación personal".

Ahora bien, si la planificación es el proceso de organizar los objetivos, métodos y materiales de los contenidos en tiempo y espacio, entonces, a través de este estudio pudo evidenciarse que los docentes encuestados presentan grandes dificultades en este proceso, lo que lleva a unas clases poco creativas, dinámicas, motivadoras, aspectos que impiden a los estudiantes apropiarse de los conocimientos necesarios para lograr competencias en el área de matemáticas. En este sentido, se puede afirmar que la planificación de los docentes estudiados es improvisada.

Lo antes expuesto contradice lo planteado por Matus (1987 como se citó en Samper, 2012), quien expresa que planificar es un proceso de análisis y discusión que conlleva al docente a reflexionar y fijar una dirección que guíe la transformación hacia la situación deseada. Es decir, docente debe interpretar la planificación en dos vertientes: a) como proceso, al llevar a cabo la acción de planificación y b) como producto al convertirse esta en la guía didáctica que orienta y facilita su trabajo como el del alumno.

Con relación a la facilitación, los resultados indicaron que los docentes de la institución en referencia desarrollan medianamente este proceso, afirmación que tiene su asidero en la valoración obtenida por la mediana (20 puntos de un máximo de 50 puntos), es decir, las técnicas, estrategias y explicaciones en clase limitan las acciones del docente para lograr que los alumnos sean unos seres capaces de razonar, pensar, discutir, y manejar la información que se les presente para su propio beneficio y del mundo que los rodea.

Estos resultados reflejan la no utilización de variados recursos por parte de los docentes en los procesos de enseñanza, lo cual genera apatía de los estudiantes y contradice el planteamiento de Quintero (2012) cuando expresa que la facilitación permite definir una secuencia de acciones centradas en los participantes, promover la construcción colectiva y generar reflexiones para aplicar lo aprendido en la transformación de la realidad, implica además, diseñar una serie de objetivos, contenidos, actividades y dinámicas para promover la participación colectiva. 
En razón de lo expuesto, se infiere que los docentes solo desarrollan parte de los procesos de enseñanza en su praxis docente

Finalmente, en la evaluación, se reflejó que medianamente los docentes observados desarrollan en sus clases procesos de evaluación de acuerdo con los tipos de contenidos y los momentos de la clase, además, mostraron dificultades sobre los tipos de técnicas y el contenido a evaluar. Asimismo, los maestros no realizan una evaluación diagnóstica que les permita determinar los conocimientos previos que posee el estudiante, a fin de tomar decisiones sobre las herramientas a manejas al momento de la clase.

Los resultados antes expuestos contravienen lo planteado por Tobón (2010, p. 36) al afirmar que "la evaluación es un procedimiento para determinar los avances de los estudiantes en la obtención de los conocimientos establecidos en el currículo".

\section{CONCLUSIONES}

0 on relación al proceso de enseñanza se concluye que los docentes estudiados, desarrollan bajos procesos de enseñanza, lo cual significa que tienen serias dificultades para abordar las acciones que conllevan a su práctica educativa que requiere de la formulación de logros u objetivos claros y precisos así como la selección de técnica y actividades didácticas de acuerdo a los contenidos y logros a alcanzar. Es medular entender que los objetivos de la enseñanza van más allá de los contenidos curriculares, se quiere desarrollar personas bien integradas, que sean capaces de asumir un rol responsable, independiente y activo en la sociedad.

Con respecto a las sinergias que midieron el proceso de enseñanza se concluye que la de menor puntaje fue la planificación, lo cual indica que los docentes realizan esta práctica de manera improvisada, sin tomar en cuenta los aspectos básicos de una planificación educativa dirigida a definir los logros de aprendizaje de acuerdo a las necesidades del estudiante y a los contenidos a enseñar. A partir de estos resultados se plantea que quizás los maestros tienen deficiencias en su formación pedagógica referida a cómo hacer una planificación eficiente y eficaz. 
Las sinergias facilitación y evaluación se ubicaron en una categoría de mediana ante lo cual se concluye que los docentes de matemáticas del estudio solo desarrollan sus clases con una explicación oral discursiva, con recursos instruccionales limitados al tablero y al cuaderno de ejercicios. Con respecto a las técnicas y actividades didácticas estas se centran en la resolución de ejercicios en las clases sin incentivar el pensamiento crítico y reflexivo. Similar situación sucede con la evaluación que se limita a pruebas escritas con énfasis en la búsqueda de que el estudiante refleje con exactitud el procedimiento o proceso que el docente enseño.

\section{REFERENCIAS}

Arce, R. (2012). Facilitación de procesos sociales. Manual para facilitar acuerdos sociales en el marco de la gobernabilidad democrática intercultural. Bolivia: Tarea Asociación Gráfica Educativa. Disponible en: https://www.servindi.org/pdf/facilitacion_procesos_sociales.pdf

Arnold, R. (2016). Didáctica de facilitación - El cambio de la orientación "input" hacia el "outcome" para el desarrollo de competencias. Revista MetaacciónMAGAZINE, (7) Disponible en: https://www.metaaccion.com/images/pdf/583481037d81bpractico-57-didactica-de-facilitacion.pdf

Calvo, G., Rendón, D. y Rojas, L. (2004). Un diagnóstico de la formación docente en Colombia. Red Académica, (47). Universidad Pedagógica Nacional. Colombia. Disponible en: https://revistas.pedagogica.edu.co/index.php/RCE/article/view/ $5519 / 4546$

Camacho, I. (2014). La evaluación con rostro humano. Edit. SignoS, Ediciones y Comunicación, C.A

Cañal, P., Lledó, A., Pozuelos, F. y Travé, G. (1997). Investigar en la escuela: elementos para una enseñanza alternativa. Sevilla: Díada

Díaz, F. y Hernández, G. (1998). Estrategias de enseñanza para la promoción de aprendizajes significativos" en Estrategias docentes para un aprendizaje significativo. Una Interpretación constructivista. México: McGraw-Hill

Ercolino, A. y Morales, J. (2006). Concepciones previas del profesorado para la toma de decisiones en la planificación de la enseñanza. Caso Departamento de Inglés de la Universidad Metropolitana. Cuadernos Unimetanos (6). Universidad de Sevilla. 
Feldman, R. (2010. Psicología con aplicaciones en países de habla hispana. 8va Edic. México: McGraw-Hill/Interamericana Editores, S.A. DE C.V

Flórez, R. (2002). Evaluación pedagógica y cognición. Colombia: Editorial Nomos S.A

Freire, P. (1973). Pedagogía del oprimido. México: Siglo Veintiuno Editores

García, B., Coronado, A. y Montealegre, L. (2011). Formación y desarrollo de competencias matemáticas: una perspectiva teórica en la didáctica de las matemáticas Revista Educación y Pedagogía, 23 (59)

Godino, J., Batanero, C., Font, V. (2003). Fundamentos de la enseñanza y el aprendizaje de las matemáticas para maestros. Matemáticas y su Didáctica para Maestros. Proyecto EdumatMaestros. $\quad$ https://www.ugr.es/ jgodino/edumatmaestros/manual/1_Fundamentos.pdf

Hirst, P. (1977): "Qué es enseñar?", R.S. Peters (ed.): Filosofía de la educación. México: F.C.E., 295-323

López, B. y Hinojosa, E. (2011). Evaluación del Aprendizaje. Alternativas y nuevos desarrollos. México: Editorial Trillas

Ministerio de Educación Nacional -MEN (2006). Estándares Básicos de Competencias en Lenguaje, Matemáticas, Ciencias y Ciudadanas. Colombia: Imprenta Nacional de Colombia.. Recuperado de https://www.mineducacion.gov.co/1759/articles340021 recurso 1.pdf

Ministerio de Educación Nacional -MEN (1998). Lineamientos curriculares Matemáticas. Recuperado de https://www.mineducacion.gov.co/1621/articles89869 archivo_pdf9.pdf

Ministerio de Educación Nacional -MEN (1994). Ley 115 de 1994 Ley General de Educación. Disponible en: https://www.mineducacion.gov.co/1759/articles321725 archivo pdf asignacion funciones UNALDES.pdf

Navarro, $\bar{R}$., Rodríguez, M., y Barcia, M. ${ }^{-}$(2011). Didáctica y currículum para el desarrollo profesional docente. Madrid: Dykinson, S.L

Ochoa, J. y Ochoa, S. (2016). Aprendizaje y Facilitación. Quito, Ecuador: CODEU.

Oficina Regional de Educación para América Latina y el Caribe Orealc/Unesco (2014). Enseñanza y aprendizaje lograr la calidad para todos. Una mirada sobre América Latina y el Caribe. 1era. Edic. Chile: graficAnimado. Recuperado de http://www.unesco.org/new/fileadmin/MULTIMEDIA/FIELD/Sa ntiago/pdf/Mirada-regional-Informe-EPT-2013-2014.pdf 
Ortiz, A. (2009). Currículo y evaluación. ¿Cómo preparar, desarrollar y evaluar clases de calidad?. Santa Marta, Colombia: UNIMAGDALENA.

Padilla, J., Rincón, D. y Lagos, J. (2016). La poiesis en la facilitación del aprendizaje para el uso de las TIC en Educación Superior. Revista Academia y Virtualidad

Pozo, J y Gómez, M. (2001). Aprender y enseñar ciencia. Del conocimiento cotidiano al conocimiento científico.3ra. Edic. Madrid: Ediciones Morata, S.L

Quesada, R. (2001). Guía para evaluar el aprendizaje teórico y práctico. México: Editorial Limusa

Quintero, G. (2012).Diseño de procesos de facilitación de aprendizaje: Compartiendo un método. Recuperado de http://www.facilitarteprocesos.com.ve/wpcontent/uploads/2016/09/1Diseno-Procesos-FacilitacionAprendizaje.pdf

Resolución 2343 de 1996. [Ministerio de Educación Nacional]. Por la cual se adopta un diseño de lineamientos generales de los procesos curriculares del servicio público educativo y se establecen los indicadores de logros curriculares para la educación formal. 5 de junio de 1996. Recuperado de https://repository.usta.edu.co/bitstream/handle/11634/280/RES OLUCION_2343_DE_JUNIO_5_DE_1996.pdf?sequence=21\&isAl lowed $=\mathrm{y}$

Saber (2018). [Gobierno de Boyacá]. Educación para la creatividad y la vida. Histórico de pruebas Saber $3^{\circ}, 5^{\circ}, 9^{\circ}$ y 11ํㄹ 2012-217. Recuperado de: http://sedboyaca.gov.co/wpcontent/uploads/2018/04/20180420-historico-pruebas-saber2012-2017.pdf

Salcedo, H. (2011). Los objetivos y su importancia para el proceso de enseñanza-aprendizaje. Revista de Pedagogía, vol. XXXII,(91), 2011, pp. 113-130. Universidad Central de Venezuela. Caracas, Venezuela

Samper, A. (2012). La Planificación de la Educación como una práctica compleja. 2da edic. Universidad Nacional de San Luis. Argentina: Ediciones LAE

Santos, Miguel; (1993). La evaluación: un proceso de diálogo, comprensión y mejora. Málaga. Recuperado de file:///C:/Users/HP\%20PORTATIL/Desktop/LA\%20EVALUACION \%20U\%20PROCESO\%20DE\%20DIALOGO.pdf

Solar, H., García, B., Rojas, F. y Coronado, A. (2014). Propuesta de un modelo de competencia matemática como articulador entre el currículo, la formación de profesores y el aprendizaje de los estudiantes. Educación Matemática. Vol. 2. (2). México. Recuperado de http://www.scielo.org.mx/scielo.php?script=sci arttext\&pid $=$

S1665-58262014000200002 
Tobón, S. (2010). Formación integral y competencias. Pensamiento complejo, currículo, didáctica y educación. Bogotá: ECOE

Unesco. (2010). Educación. Recuperado de https://es.unesco.org/creativity/sites/creativity/files/digitallibrary/cdis/Educacion.pdf

Unesco. (2015). Estrategia de Educación de la Unesco 2014-2021. Recuperado de https://unesdoc.unesco.org/ark:/48223/ pf0000231288 spa

Vygotsky, L. (1978). El desarrollo de los procesos psicológicos superiores. Edición al ciudadano. 1era edición. Barcelona: Editorial Crítica. Recuperado de http://www.terras.edu.ar /biblioteca/6/TA_Vygotzky_Unidad_1.pdf 